\title{
Binary interaction effects: predictions and observations
}

\author{
Gloria Koenigsberger, Edmundo Moreno, Jorge Cantó, \\ and Alejandro C. Raga \\ Instituto de Astronomía, UNAM, México
}

\begin{abstract}
Observations and predictions of interactions in close and massive binary systems are summarized. Tidal interactions are analyzed using a model which predicts the response of the stellar surface to the external gravitational force of the companion. The possible role of tidal interactions in determining the peculiar behavior of systems like HD 5980 and $\eta$ Carinae is discussed.
\end{abstract}

\section{Introduction}

The interaction effects in massive binary systems are numerous and they can significantly alter the observable characteristics of the system with respect to the simple sum of the characteristics of its individual stars. Near the stars, gravitational, radiative and wind-wind collision effects dominate. Far from the stars, circumbinary wind structures develop. As a star evolves, some of its properties change, and thus the interaction effects evolve as well.

HD 5980 is a peculiar WR+O supergiant binary system in the Small Magellanic Cloud which recently underwent a series of LBV-type eruptive events. These eruptions were preceded by significant changes in the wind structure of (at least) one of the two massive members of the 19.3-day period system. $\eta$ Carinae is now believed to be an eccentric binary (Damineli et al. 1997), and though its eruptive behavior is more extreme than that of HD 5980, both systems may have many characterstics in common. Descriptions of HD 5980 and $\eta$ Car can be found in the two corresponding sections containing papers devoted to them in Nota \& Lamers (1997).

In the case of HD 5980, the radius of the erupting star as well as its mass loss rate increased systematically in a $\sim 20$ year timespan, and this star exceeded the dimensions of its classical Roche surface (Koenigsberger et al. 1998a). As the radius of the erupting star increased, it became more sensitive to the tidal forces exerted by the gravitational field of the companion, and it is these tidal forces which have been suggested as the mechanism responsible, at least in part, for triggering the unusual eruptive behavior of HD 5980 (Koenigsberger \& Moreno 1997).

\section{Wind-wind collisions, radiative braking and wind eclipses}

Over the recent years, significant effort has been devoted to the modelling and the observations of wind-wind collisions (WWC) in the WR+O binary systems. Excellent reviews and new developments are presented elsewhere in these Proceedings. A WWC model for HD 5980 is discussed by Moffat et al. (1998). 
There is, however, some debate regarding the importance of the WWC region in terms of its contribution to the emission line spectrum at optical and UV wavelengths. In the case of HD 5980, Moffat et al. (1998) have argued that a very significant contribution to the emission lines arises in the WWC region. However, Koenigsberger et al. (1998a) pointed out that the non-dereddened energy $\left(3 \times 10^{37} \mathrm{ergs} \mathrm{cm}^{-2} \mathrm{~s}^{-1}\right)$ contained in just one emission line, He II $1640 \AA$, is an order of magnitude larger than the maximum kinetic energy of the WWC that can be thermalized. Thus, a better understanding of the line-emitting characteristics of the WWC zone is needed in order to reliably extract the valuable information contained in the emission-line profiles in systems such as HD 5980. The WWC can clearly, however, be responsible for optical and UV line-profile variability, even if the shock zone does not emit significantly in these lines. The shock cone which winds around the star with the weaker wind (see Figure 3 of Stevens \& Pittard, these Proceedings) produces a 'hole' in the WR wind, that changes its position with respect to the observer as a function of orbital phase. This effect contributes to the line-profile variability in lines which are formed in the region of the WR wind affected by the shock cone's presence (Lloyd \& Stickland, these Proceedings).

It is interesting to point out that in HD 5980 the momentum flux ratio of the winds of the two stars has changed over the past $\sim 20$ years ( $c f$. Moffat et al. 1998; Koenigsberger et al. 1998a), thus changing the characteristics of the WWC region. This provides a unique opportunity for studying WWC phenomena under evolving stellar wind characteristics.

Relevant to the nature of the wind-wind collision is the radiative braking of the wind of one of the stars by the continuum emission of the other one, an effect which is predicted to be active in binary systems such as V444 Cyg (Gayley et al. 1997). Radiative braking changes the structure of the shock formed by the colliding winds. Observational evidence for this effect in V444 Cyg $(\mathrm{WN} 5+\mathrm{O} 6 \mathrm{~V})$ is the reduced speed of the WR-star wind in the direction of the Ostar companion in those regions of the wind closer to the O-star (Koenigsberger 1990; Marchenko et al. 1997).

Wind or atmospheric eclipses, also referred to as wind occultations, were first analyzed by Münch (1950) and Khaliullin \& Cherepashchuk (1976). Wind eclipses occur when the continuum emitting luminous stellar disk of a companion star is occulted by a column of wind material which lies along the line of sight to the observer, and which belongs to the Wolf-Rayet star. Under these conditions, continuum emission from the companion star is absorbed or scattered by the WR wind. Because the opacity of lines arising from permitted transitions is, in general, larger than that of continuum processes, the effect is easiest to observe in the WR emission lines. By modelling the emission line profile variations which occur when different columns of wind material are projected onto the companion star, the velocity and density laws of the WR stellar wind can be derived. An application of this method to V444 Cyg has led to the conclusion that the wind in the WR star undergoes an acceleration over several stellar radii (Auer \& Koenigsberger 1994), thus implying a much slower acceleration in the innermost portions of the wind than that predicted by the standard $\beta$-velocity law. Recent observational evidence for large $\beta$-values in several WRs has been presented by Lepine \& Moffat (1999). The wind eclipses are 
also responsible for phase-dependent flux variations in several wavelength bands between $\lambda \lambda 1270-1700 \AA$, corresponding to the 'forests' of Fe IV, Fe V and Fe VI lines. The variations observed in these various bands at different orbital phases provide observational evidence (Koenigsberger 1990) for the ionization stratification in the WR star winds. The role of this stratification in governing the wind velocity structure is stressed by Schmutz (1995) and new developments reviewed by Crowther (these Proceedings). Other results derived using the wind-eclipse model are presented by Schweickardt et al. (these Proceedings).

Wind eclipse effects in HD 5980 are evident in the spectra obtained by IUE in 1986 (Moffat et al. 1989). From these data it was concluded that the wind of the WN4 star contained a large column density of N IV ions out to distances larger than $50 \mathrm{R}_{\odot}$, while the wind of the companion was dense enough to produce observable effects in C IV $1550 \AA$. However, a determination of the wind structures in HD 5980 using the eclipse model is hampered by: (1) difficulties in isolating, in the observed spectrum, the emission which arises from the wind-wind collision region; and (2) uncertainties in the values of the orbital parameters.

\section{Spiral shock structures and circumbinary disks}

Consider a binary system in which only one of the stars has a wind and in which the gravitational field can be neglected. This scenario is valid for relatively large orbital separations or very large wind velocities. The orbital motion induces the formation of a large scale spiral shock structure in the wind (see Figure 1 of Cantó et al. these Proceedings). Cantó et al. (1999) present an analytic formulation of the problem which provides the distance from the star at which the shock wave appears, and also the topology of the spiral shock structure. These surfaces wind around the binary system, and the entire shock pattern rotates with a period equal to the orbital period. Numerical simulations show that the analytic solution reflects the true shock structure of the wind, and allow precise predictions of the shock strengths (Cantó et al. these Proceedings).

As with the co-rotating interaction regions (CIR) model for single stars, the three-dimensional spiral structure in the binary systems could be responsible for the appearance of discrete absorption components (DACs) in the spectra of the binary systems. A recurrence time equal to the orbital period is predicted for the behavior of these features.

Contrasting with the case described above is the case in which the gravitational fields of both stars are important enough to affect the stellar wind of one of the stars. This scenario applies, for example, to close binary systems containing an LBV star, in which the wind is relatively slow. Numerical experiments in which the trajectories of wind particles are computed indicate that a significant fraction of these particles are deflected towards the orbital plane of the binary system (Koenigsberger et al. 1999), similar to what has been found to occur in low-mass, common envelope binary systems (see the review by Iben \& Livio 1993). This leads to the formation of a large scale, expanding circumbinary disk structure.

We note that in close binaries in which both stars have winds; the wind-wind collision contributes to lower the expansion velocity of the particles involved in the collision, and thus renders them much more susceptible to being deflected 
by the gravitational potential of the system. Radiation pressure from the companion acts in a similar fashion.

In the case of HD 5980, the formation of a circumbinary disk structure is predicted to have occurred at the time of the eruptive events, when wind velocities were $v_{\text {wind }}<600 \mathrm{~km} \mathrm{~s}^{-1}$. As the wind velocities increased once again, these fast winds would have reached and interacted with the more slowly expanding disk. This scenario may also be applicable to $\eta$ Carinae as a mechanism for forming the equatorial disk-like structure of the homunculus. Other mechanisms which have been proposed involve stellar rotation (see Garcia-Segura et al. these Proceedings), such as the wind-compressed model of Bjorkman \& Cassinelli (1996), although it is now unclear whether such a mechanism actually does produce a dense equatorial disk (cf. Owocki 1999).

\section{Tidally induced oscillations}

In a close binary system, the gravitational field of each of the stars acts as an outer perturbing force on its companion, and through the resulting tidal interaction the stellar surfaces are deformed with respect to their unperturbed shape. The tidal interactions have been shown to be a mechanism by which a binary system tries to reach an equilibrium state. A binary system is defined to be in an equilibrium state when the equators of both stars lie in a plane which coincides with the orbital plane, the orbits are circular, and the stellar rotation periods equal the orbital period. The circularization of the orbits and the corotation of the stellar components are achieved by the exchange of orbital and rotational angular momentum and energy, through the torques exerted by the tides. The exact mechanisms by which this transfer takes place differ in stars which have convective envelopes from those with radiative envelopes (Zahn 1977, 1989; see also Goldreich \& Nicholson 1989).

Although the primary motivation for the analysis of the tidal interactions has been the understanding of the evolution of binary system orbital parameters, the possible role of tidal interactions in triggering eruptions in systems such as HD 5980 and $\eta$ Car makes their analysis from this standpoint relevant (Koenigsberger \& Moreno 1997).

We have developed a model to study the detailed effects on the stellar surface of a star in a binary system, due to the presence of its companion's gravitational field (Moreno \& Koenigsberger 1999, in preparation). The model allows an analysis of the dependence of these effects on the binary system parameters, and on the viscous forces of the gas. The approach is to solve the equations of motion for each of 100 surface elements chosen to lie along the equator of the star, in the orbital plane. The other forces included in the calculation, in addition to the gravitational field of both stars, are those arising from gas pressure and viscosity.

\subsection{Oscillations on the time-scale of the orbital period}

The calculations for a model binary system in a moderately eccentric $(e=0.3)$ orbit and $P_{\text {orb }}=16.5$ days, are presented in Figures 1 and 2 . The other system parameters which remain constant throughout these calculations are: the orbital semi-mayor axis, $a=115 \mathrm{R}_{\odot}$; the stellar masses, $M_{1}=46 \mathrm{M}_{\odot}$, and $M_{2}=30 \mathrm{M}_{\odot}$; the radius of the companion star, $R_{2}=15 \mathrm{R}_{\odot}$. Two cases for the stellar radius of 


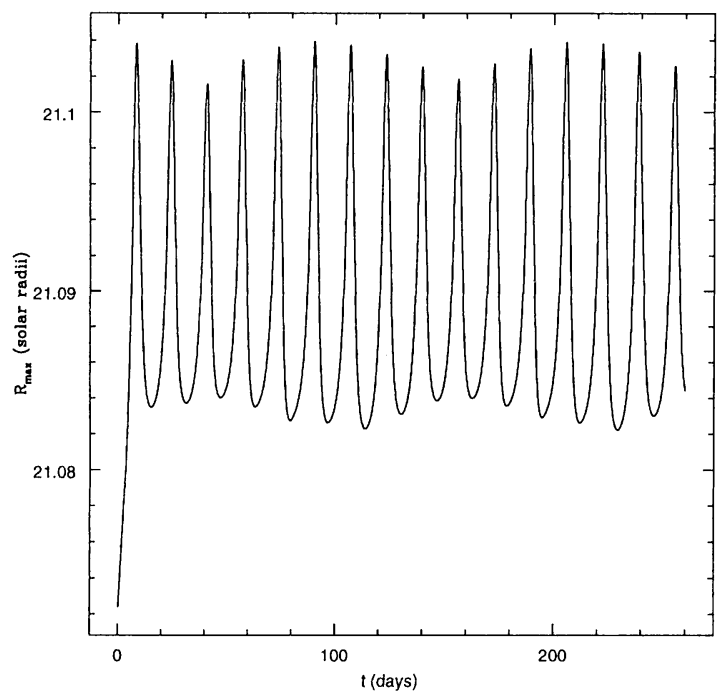

Figure 1. Oscillations in the maximum stellar radius in a moderately eccentric (co-rotating at apastron) binary system. The equilibrium radius of the perturbed star is $21 \mathrm{R}_{\odot}$.

the erupting component are analyzed: $R_{1}=21 \mathrm{R}_{\odot}$ and $R_{1}=35 \mathrm{R}_{\odot}$. The calculation is started at apastron, with the perturbed star, star 1 , having a spherical shape and with a rotation angular velocity equal to the orbital angular velocity (i.e., co-rotating at this orbital phase). The star very rapidly responds to the external force and deforms its shape adopting a prolate spheroidal shape, with the semi-major axis oriented in the general direction of the companion. As periastron is approached, the degree of deformation increases. The changing dimensions of the star are represented by the parameter $R_{\max }$, the maximum stellar radius achieved at a given time. In these calculations, the azimuth angle between the orbital semi-mayor axis and the orientation of $R_{\max }$ is between $-30^{\circ}$ and $-10^{\circ}$; that is, for all orbital phases, the tidal bulge is not oriented directly towards the companion, but 'lags behind', lagging much more near periastron $\left(-30^{\circ}\right)$ than at apastron.

Figures 1 and 2 show the variations of $R_{\max }$ from apastron (minimum value) to periastron (maximum values). The maximum value of $R_{\max }$ is achieved slightly after periastron passage. The oscillation amplitudes depend on the value of the perturbed star's radius, and on the viscous forces, $\nu$, of the surface gas, being greater for larger stellar radii and smaller viscosities. Under the assumption of very small viscous forces, the response of the stellar layers is instantaneous and the amplitude of the oscillations is large. However, for the small viscosity models at large stellar radii, the solution of the equations of motion for the surface elements indicates that these elements begin to overlap and cross over each other. We tentatively associate this effect with turbulence in the surface layers. This aspect of the model requires further analysis, but it is interesting to note that the values of the radius and viscosity at which this 'turbulence' appears, depend on the rotation rate of star 1. For the present, we 


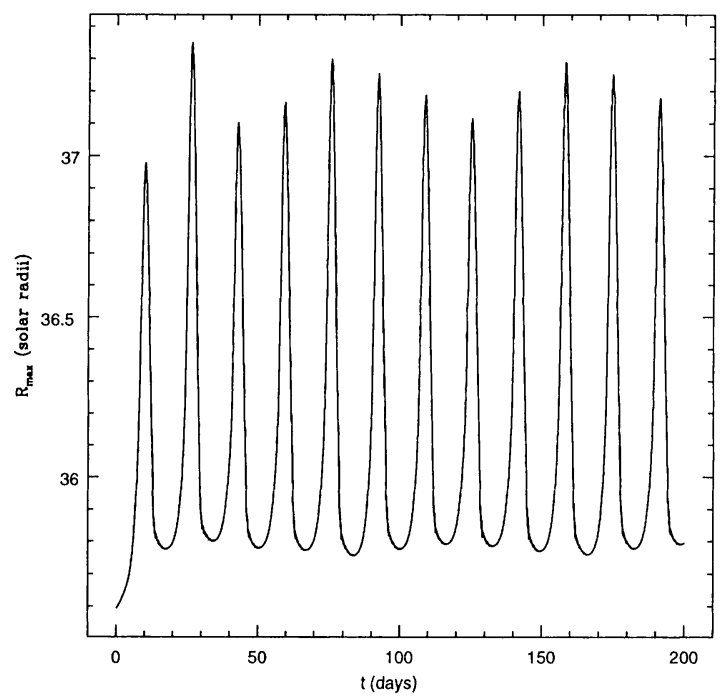

Figure 2. Similar to Figure 1, but with $R_{1}=35 \mathrm{R}_{\odot}$.

set the value of $\nu$ to be just large enough to avoid this effect. When larger values of $\nu$ are used, for a fixed set of binary system parameters, the amplitude of the oscillations is reduced. In the example illustrated in Figure $1, \nu=0.1 \mathrm{R}_{\odot}^{2} \mathrm{day}^{-1}$ and $R_{1}=21 \mathrm{R}_{\odot}$, and the oscillation amplitude is smaller than $0.05 \mathrm{R}_{\odot}$, while in Figure 2, $\nu=0.7 \mathrm{R}_{\odot}^{2}$ day $^{-1}$ and $R_{1}=35 \mathrm{R}_{\odot}$, and the amplitude is larger than $1 \mathrm{R}_{\odot}$. Here $\nu=1.0 \mathrm{R}_{\odot}^{2}$ day $^{-1}=5.6 \times 10^{16} \mathrm{~cm}^{2} \mathrm{~s}^{-1}$, is the coefficient of kinematical viscosity.

We note that for the binary parameters we are considering, for $R_{1}>35 \mathrm{R}_{\odot}$, the value of $R_{\max }$ at periastron exceeds the dimensions of the classical Roche surface, and thus large scale outflows from star 1 can be envisioned, first only at periastron passage, but as the stellar radius grows, the large scale outflow should occur throughout the orbital cycle. Thus, the prediction for HD 5980 is that the major eruption observed in 1994 should have been preceded by a series of shorter duration enhanced mass loss events, triggered by the periastron passages, some of these perhaps producing a temporary contraction of star 1 , followed by its subsequent expansion. Possible observational evidence in support of these episodic enhanced mass-loss events are the non-orbital phase related variations reported by Barbá et al. (1997), and the smaller eruptive events observed to occur one year prior to the 1994 major eruption (see the light curve shown in Breysacher 1997). The initial cause of the expansion of the star leading it into this situation remains, however, as yet unclear. If the mechanism resulting in the expansion of star 1 is still active, then future eruptions are expected.

\subsection{Surface oscillations on short time-scales}

Due to the elastic properties of the stellar material and to the driving force exerted by a companion's gravitational field, a star also undergoes short time-scale oscillations superposed on the oscillations which occur on the orbital time-scale. The description of these forced oscillations has been made by Zahn (1977), and 
Kumar et al. (1995), among others and an example from our model calculations is illustrated in Figure 3 , for $R_{1}=35 \mathrm{R}_{\odot}$ and $\nu=0.5 \mathrm{R}_{\odot}^{2}$ day $^{-1}$. The computation was started at apastron with star 1 rotating with a value of angular velocity equal to one half the value of the orbital angular velocity; i.e., this is a non-corotating case. The short time-scale oscillations are found to be more pronounced after periastron passage, and their amplitude is strongly dependent on the value of the viscosity coefficient, with smaller viscosities allowing larger oscillation amplitudes.

Note that the time-scale of the predicted tidal oscillations is $\sim 0.3$ day. Curiously, this is very similar to the period of 0.25 day recently reported for HD 5980 by Sterken \& Breysacher (1997) (see also Marchenko et al. 1994, who report $P=0.36$ day for $\mathrm{V} 444 \mathrm{Cyg}$ ). Although it is tempting to speculate that these micro-variations are due to the tidal forces in the binary system, there are discrepancies between the observations and the model predictions: a) the data indicate that the oscillations persist at nearly the same amplitude throughout the orbital cycle, while our model predicts stronger effects after periastron passage; and b) assuming that the photometric variability is due to radial pulsations, the amplitude of the radius' variation is too large $\left(1.5 \mathrm{R}_{\odot}\right.$, Schweickhardt \& Schmutz, these Proceedings), compared with the predictions shown in Figure 3 for the superposed, short time-scale variations. An opposite problem is found in the case of the very eccentric binary system $\iota$ Ori, where Gies et al. (1996) failed to find, in their observations, the effects predicted to occur due to the tidal oscillations using the model of Kumar et al. (1995). The cause of these discrepancies needs to be understood.

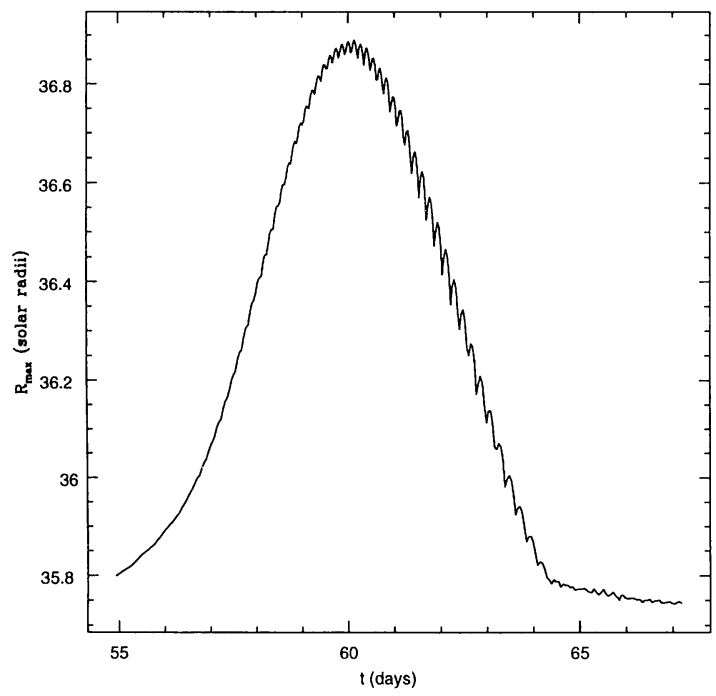

Figure 3. Short time-scale surface oscillations superposed on the variations which occur on the orbital time-scale. In this model, $\nu=0.5 \mathrm{R}_{\odot}^{2} \mathrm{day}^{-1}$, $R_{1}=35 \mathrm{R}_{\odot}$, and the perturbed star is not in co-rotation. 


\section{Concluding reflections}

We have reviewed some of the predictions and observational evidence for various of the interaction effects in binary systems, focusing on HD 5980, a system in which nearly all imaginable interaction effects are possible. Some of these effects have far reaching consequences on the morphology of the interstellar matter ejected by the star and on the evolution of the two stars in the system. In particular, the question of the role of tidal forces in the formation of WR stars in binary systems, and their wind characteristics requires further analysis. Some of the questions which arise in this context are: (1) What is the role that tidally induced oscillations play in mixing of surface layers with interior, chemically altered layers of the star? (2) Do these oscillations contribute significantly towards driving the stellar winds and producing non-spherically symmetric wind structures? (3) Do they serve to drive the formation of wind-embedded shocks, thus enhancing X-ray emission rates with respect to those of single stars? (4) Do they play an important role in inducing eruptive, LBV-type events, such as we have observed in HD 5980 and $\eta$ Carinae or other eruptive phenomena in eccentric orbit WC stars such as WR 140 ?

Acknowledgments. Support from UNAM/DGAPA through the grants IN109496 and IN109596 is gratefully acknowledged. GK thanks S. Owocki, A. Maeder and N. Langer for may helpful discussions.

\section{References}

Auer, L.H., Koenigsberger, G. 1994, ApJ 436, 859

Barbá, R., Niemela, V.S., Morrell, N.I. 1997, in: A. Nota \& H. Lamers (eds.), Luminous Blue Variables: Massive Stars in Transition, ASP-CS 120, 238

Bjorkman, J.E., Cassinelli, J.E. 1996, ApJ 409, 429

Breysacher, J. 1997, in: A. Nota \& H. Lamers (eds.), Luminous Blue Variables: Massive Stars in Transition, ASP-CS 120, 215

Cantó, J., Raga, A., D’Alessio, P. 1999, MNRAS submitted

Damineli, A., Conti, P.S., Lopes, D.F. 1997, New Astronomy 2, 107

Gayley, K.G., Owocki, S.P., Cranmer, S.R. 1997, ApJ, 475, 786

Gies, D.R., Barry, K.J., Bagnuolo, W.G., Sowers, J., Thaller, M.L. 1996, ApJ 469, 884

Goldreich, P., Nicholson, P.D. 1989, ApJ 342, 1079

Iben, I., Livio, M. 1993, PASP 105, 1373

Khaliullin, Kh.F., Cherepashchuk, A.M. 1976, Sov. Astron. 20, 186

Koenigsberger, G. 1990, A\&A 235, 282

Koenigsberger, G., Moreno E. 1997, in: A. Nota \& H. Lamers (eds.), Luminous Blue Variables: Massive Stars in Transition, ASP-CS 120, 332

Koenigsberger, G., Auer, L.H., Georgiev, L., Guinan, E. 1998a, ApJ 496, 934

Koenigsberger, G., Peña, M., Schmutz, W., Ayala, S. 1998b, ApJ 499, 889

Koenigsberger, G., Moreno, E. and Cantó, J. 1999, in: B. Wolf, A.W. Fullerton \& O.

Stahl (eds.) Variable and Non-Spherical Stellar Winds in Luminous Hot Stars,

Proc. IAU Coll. 169, Lecture Notes in Physics ..., 260

Kumar, P., Ao, C.O., Quataert, E.J. 1995, ApJ 449, 294

Marchenko, S., Moffat, A.F.J., Koenigsberger, G. 1994, ApJ 422, 810

Marchenko, S., Moffat, A.F.J., Eenens, P. et al. 1997, ApJ 485, 826 
Moffat, A.F.J., Koenigsberger, G., Auer, L.H. 1989, ApJ 344, 734

Moffat, A.F.J., Marchenko, S.V., Bartzakos, P. et al. 1998, ApJ 497, 896

Münch, G. 1950, ApJ 112, 266

Nota, A., Lamers, H. (eds.) 1997, Luminous Blue Variables: Massive Stars in Transition, ASP-CS 120, 215

Owocki, S.P. 1999, in: B. Wolf, A.W. Fullerton \& O. Stahl (eds.) Variable and NonSpherical Stellar Winds in Luminous Hot Stars, Proc. IAU Coll. 169, Lecture Notes in Physics ..., 294

Schmutz, W. 1995, in: K.A. van der Hucht \& P.M. Williams (eds.), Wolf-Rayet Stars: Binaries, Colliding Winds, Evolution, Proc. IAU Symp. No. 193 (Dordrecht: Kluwer), p. 127

Sterken, C. \& Breysacher, J. 1997, A\&A 328, 269

Zahn, J.-P. 1977, A\&A 57, 383

Zahn, J.-P. 1989, A\&A 220, 112

\section{Discussion}

Heap: Is it possible that the emission blip at $v=-800 \mathrm{~km} \mathrm{~s}^{-1}$ in the spectrum of HD 5980 is an ionization hole created in some way by the WN3-4 companion?

Koenigsberger: The true continuum level would have to be very high for the S IV 1402 feature near $v=-800 \mathrm{~km} \mathrm{~s}^{-1}$ in HD 5980, visible in the IUE spectrum swP52888, to be an ionization hole. It is, however, a curious coincidence that the maximum extent of the Si IV $1400 \mathrm{P}$-Cygni lines gives a velocity of $v=-1700 \mathrm{~km} \mathrm{~s}^{-1}$ which we believe is associated with the non-erupting WN3-4 companion.

Lloyd: I have a comment about the spiral structure of the shock zone. In the IUE spectrum of WR 79 (WC7+O5-8) is a clear spectral signature which shows the shock zone is wrapped around the system. $\gamma^{2}$ Vel shows asymmetric variations which also suggest that the shock zone is wrapped around the system and other stars may show similar effects.

Zhekov: What is the ratio between the orbital velocity and the wind velocity? In fact, I do not expect any spiral shock to form if this ratio is small, since in this case a wind self-interaction is hardly expected.

Koenigsberger: One expects this ratio to be $\ll 1$. A typical figure would be $\sim 0.1$. Shocks are expected if the orbital velocity is greater than the sound speed in the wind $\left(\sim 10 \mathrm{~km} \mathrm{~s}^{-1}\right)$. Since $v_{\text {wind }} \simeq 2000 \mathrm{~km} \mathrm{~s}^{-1}$, one expect that this kind of shocks will always be formed. See Cantó et al. (these Proceedings).

Vanbeveren: The spectral type of the O-type component in HD 5980 suggests that it is very young (even close to the ZAMS). However, the WR component is H-deficient and must be several Myr old. Is there an explanation for this discrepancy?

Koenigsberger: My estimate of the high-temperature limit for the erupting star in the system has large uncertainties (but see Schweickhardt \& Schmutz, these Proceedings).

De Marco: Parameters of WR stars in binaries can be wrong (as can the classification), if the companion orbits within the wind of the primary star. This is because the WR models used to model the wind emission-lines do not take into account an obscuring/emitting object within the line-forming region 